\title{
„LGBT*I \& AGING“ für Vielfalt in der Pflege
}

Wie blicken lesbische, schwule, bisexuelle, trans* und intergeschlechtliche (LSBT*I) Senior*innen auf das Alter(n)? Welche Folgen haben Hetero- und Asexualisierung in pflegerischen Regeldiensten? Im Rahmen des IFAF-Forschungsprojekts „Gleichgeschlechtliche Lebensweisen und Pflege im Alter" (GLEPA) der ASH Berlin wurde die internationale Fachtagung „LGBT*I \& AGING - gefördert von der Deutschen Forschungsgemeinschaft (DFG) - veranstaltet.

" Zur Situation von lesbischen, schwulen, bisexuellen Senioren und älteren Trans*- und Intersex-Personen (LSBT*I) gibt es nur wenige Untersuchungen. Dabei berichten die wenigen Studien vom deutlichen Unbehagen dieser Bevölkerungsgruppen gegenüber regulären Angeboten der offenen und stationären Altenhilfe, u.a. aufgrund von befürchteten homophoben Reaktionen. Auch die GLEPA-Studie der Alice Salomon Hochschule Berlin (ASH) illustriert diese Befunde. Ergebnisse für ein verbessertes Verstehen der sozialen, gesundheitlichen und pflegerischen Bedürfnisse von LSBT*-Älteren wurden von Prof. Dr. María do Mar Castro Varela und Dr. Ralf Lottmann, beide ASH, vorgestellt und mit den Teilnehmenden diskutiert.

Dr. Martin Moerings (NL) stellte den Pink Passkey, ein Auditverfahren und Pflege-Zertifikat für ambulante und stationäre Pflege in den Niederlanden, vor. Unter anderem mittels eines „To-
leranz-Scans" werden die Einrichtungen auf ihre Offenheit gegenüber LSBT* Pflegebedürftigen und deren Inklusion geprüft - womit sie nach außen sogar für neue Marktsegmente werben können. Frédéric Lauscher vom Frankfurter Verband für Altenpflege e.V. berichtete in diesem Zusammenhang von den Erfahrungen des in 2012 übertragenen Konzepts in zwei Frankfurter Pflegeheimen. Das Resultat zeigte eine positive Resonanz nicht nur von LSB( $\left.T^{*} \mathrm{I}\right)$-Senioren, sondern von allen Bewohnern. Eine steigende Sichtbarkeit von LSBT* Pflegebedürftigen in den Einrichtungen und eine bessere Haltequote von LSB-Pflegepersonal vor dem Hintergrund eines starken Fachkräftemangels sind weitere Effekte in den beiden Frankfurter Pflegeheimen. In der anschließenden Diskussion wurden Unterschiede zwischen der Ausbildung von Pflegepersonal in den Niederlanden und in Deutschland diskutiert. Zudem wurde die Frage ge- stellt, inwieweit dieses Konzept aus einer intersektionalen Perspektive inklusiv wirken kann. Es wurde diskutiert, welche Überschneidungen und Unterschiede der Pink Passkey bzw. die Versorgung von LSBT*I Senior*innen in Bezug zur kultursensiblen Altenpflege bestehen und welche Schritte sich dafür in Forschung und Praxis ergeben.

Prof. Kathryn Almack, University of Hertfordshire, Großbritannien, stellte die Studie „The Last Outing - Exploring End of Life Experiences of Older LGBT* people" vor. Mit den Teilnehmenden wurden am Abschluss der Tagung die Ergebnisse zusammengefasst und die Notwendigkeit zukünftiger Kooperationen zu diesem Themenfeld bekräftigt. Dies sei nötig, um „weiße Felder“ in der Gerontologie, sozialen Arbeit und den Pflegewissenschaften $\mathrm{zu}$ schließen und praxisnahe Handlungsmaterialien und -forderungen weiter konkretisieren zu können.

Dr. Ralf Lottmann,

Prof. Dr. Ingrid Kollak, ASH Berlin www.ash-berlin.eu/hochschule/lehrende/professorinnen/prof-dr-ingrid-kollak/\#c5291

Quelle:

„LGBT*I \& AGING: Needs, opportunities and challenges - Lesbian, Gay, Bi, Trans* and Intersex Aging in Theory and Practice"; Berlin, 6.-7. April 2017.

\section{Fleischesser sterben früher}

\section{Der Verzehr von rotem Fleisch geht mit einem erhöhten Sterbe- risiko einher, berichten Forscher des Nationalen Krebsinstituts der USA. Sind Eisen und Nitrat schuld?}

" In der NIH-AARP Diet and Health Study mit mehr als einer halben Million Teilnehmern hatte sich eine Steigerung des Sterberisikos um $26 \%$ ergeben, wenn man das Quintil mit dem höchsten Konsum von rotem Fleisch $(67,5 \mathrm{~g} / 1.000 \mathrm{kcal})$ mit dem niedrigsten (9,3 g/1.000 kcal) verglich. Auch die Mortalität für Krebs, Herzerkrankungen, Schlaganfall, Atemwegsleiden,
Diabetes, Infektionen sowie Nierenund Leberkrankheiten war bei den Rotfleischessern erhöht.

Besonders nachteilig war der Verzehr von verarbeitetem rotem Fleisch, was die Autoren durch das enthaltene Nitrat erklären: Etwa die Hälfte der Erhöhung der Gesamtmortalität ging darauf zurück. Bei nicht verarbeitetem rotem Fleisch war das Häm-Eisen für
21\% der erhöhten Gesamtmortalität und rund ein Drittel der Zunahme bei den Krebsfällen verantwortlich.

Quelle:

MMW 10/2017 basierend auf: Etemadi A, Sinha $R$ et al. Mortality from different causes associated with meat, heme iron, nitrates, and nitrites in the NIH-AARP Diet and Health Study: population based cohort study. BMJ. 2017 May 9;357:j1957. 